\title{
Feature Set Fusion for Spoof Iris Detection
}

\author{
P. V. L. Suvarchala \\ Jawaharlal Nehru Technological University \\ Kakinada \\ Andhra Pradesh, India \\ suvarchala_pvl@yahoo.com
}

\author{
S. Srinivas Kumar \\ Department of ECE \\ Jawaharlal Nehru Technological University, Kakinada \\ Andhra Pradesh, India \\ samay_ssk2@yahoo.com
}

\begin{abstract}
Iris recognition is considered as one of the most promising noninvasive biometric systems providing automated human identification. Numerous programs, like unique ID program in India - Aadhar, include iris biometric to provide distinctive identity identification to citizens. The active area is usually captured under non ideal imaging conditions. It usually suffers from poor brightness, low contrast, blur due to camera or subject's relative movement and eyelid eyelash occlusions. Besides the technical challenges, iris recognition started facing sophisticated threats like spoof attacks. Therefore it is vital that the integrity of such large scale iris deployments must be preserved. This paper presents the development of a new spoof resistant approach which exploits the statistical dependencies of both general eye and localized iris regions in textural domain using spatial gray level dependence matrix (SGLDM), gray level run length matrix (GLRLM) and contourlets in transform domain. We did experiments on publicly available fake and lens iris image databases. Correct classification rate obtained with ATVS-FIr iris database was $100 \%$ while it was $95.63 \%$ and $\mathbf{8 8 . 8 3} \%$ with IITD spoof iris databases respectively.
\end{abstract}

Keywords-iris recognition; liveness detection; spoofing; print attacks; contact lens attacks

\section{INTRODUCTION}

Iris recognition is an extensively used biometric system by private and government agencies for its precision and trustworthiness. It has been treated as one of the most promising technologies to provide reliable human recognition [1]. Iris recognition is now an indispensable module of large scale biometric identification for financial transactions and social benefits, e.g. iris biometrics is used in the Aadhar project to provide dependable identification for millions of citizens in India [2]. As a result, the veracity of such a large scale system must also ensure to avoid likely threats such as spoofing attacks, which can cause vulnerability to the iris recognition systems. The possibility of using good quality printed iris images and eye images with contact lens can compromise the iris recognition system.

Spoofing attack techniques try to deceive the iris recognition system by presenting synthetically generated iris images printed using quality printing devices. Author in [3] stated that commercial systems can be fooled with a printout down to a resolution of $1200 \mathrm{dpi}$, an iris diameter of 75 pixels and at least $75 \%$ of the iris being visible. Latest attacks such as those detailed in [4] show more sophisticated threats rising from the usage of textured superficial lenses that present changed iris texture to the iris acquisition system which can possibly compromise the installed iris recognition systems. While colored or textured contact lenses are being worn for cosmetic reasons, it is becoming a practice for ophthalmologists to prescribe contact lens for medical reasons to correct the eye sight. Hence it is unavoidable for the recognition algorithm to find the presence of lens. In [5] it was shown that the texture details in near-infrared imaging are prominent and course and they will obfuscate the real or natural iris patterns. Detection of contact lens presence is an important step toward improving reliability of iris recognition. Author in [6] applied Fourier transform to detect the fake patterns due to textured lenses.

The hypothesis generated and tested in [7] is that, the iris detection algorithm has to first reject the instances with obfuscated patterns and allow only the soft lens and no lens cases. In the proposed method we tested the hypothesis on three databases, one of them being print attack and the others being soft lens and textured lens cases. Figure 1 shows some sample images of the real and fake (printed) iris images from ATVS-FIr a publicly available database [8]. There are 1600 fake and real images of iris (800 from each category) captured from both eyes of 50 subjects treated as 100 classes. Figure 2 shows sample images of the real and fake (cosmetic and transparent contact lens) iris images of IIIT-D Vista, a publicly available database [9] acquired from 101 subjects, 202 classes comprising of 6570 iris images.

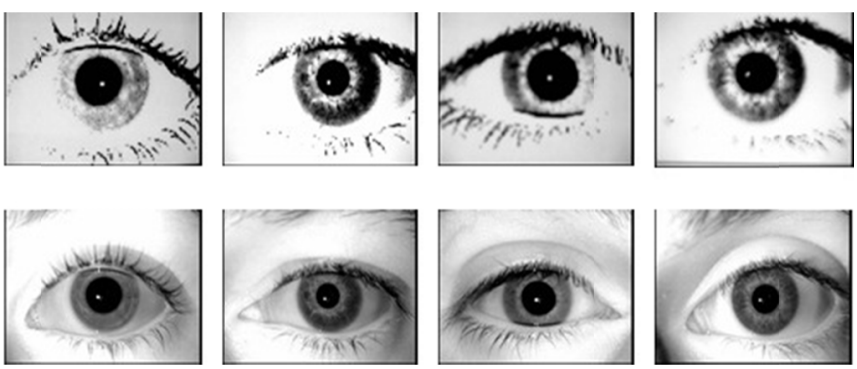

Fig. 1. Row 1: Eye images with print attack, Row 2: Original eye images from ATVS-FIr Database

The proposed spoof iris detection approach analyzes image features such as intensity distribution, coarseness and stochasticity of the iris texture and edge information which is computed from the gradient of global eye image. The developed approach also exploits the textural region of the 
entire eye image using SGLDM and GLRLM which can provide very useful descriptors. The block diagram of our approach for spoof iris detection is shown in Figure 3. The developed approach works on spoofed images with printed attacks, while sustaining recognition capability in the presence of soft (transparent) lens. In addition to textural features, we also computed transform domain features using contourlet transform. Contourlets are suitable for extracting information from images with smooth edges and contours. The classification performance of the algorithm is tested using support vector machines (SVMs).

The rest of this paper is structured as follows. In section II, feature set generation is described. In section III dataset partition and classification are presented. In section IV the experimental results are presented. In section $\mathrm{V}$ the key conclusions of the proposed method are given.
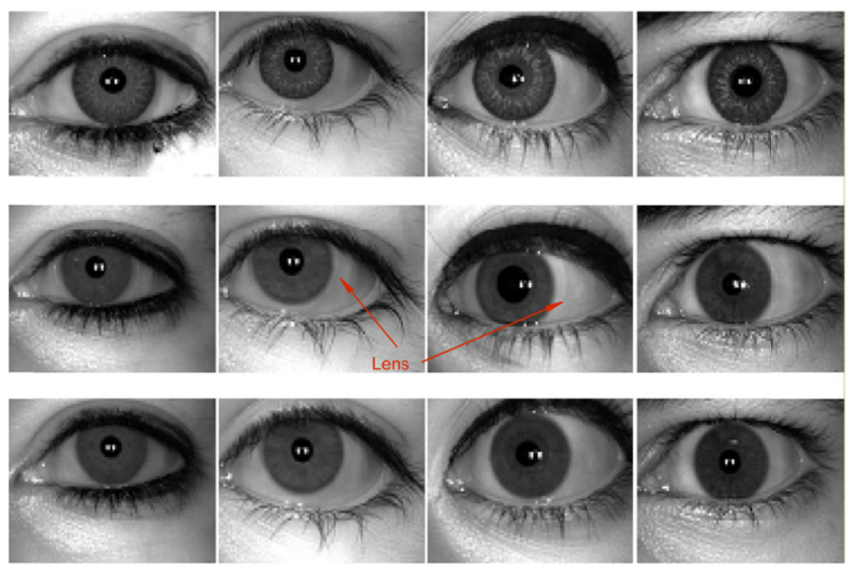

Fig. 2. Row 1: Colored contact lens images, Row 2: Transparent contact lens images, Row 3: Images with no lens from IIITD database.

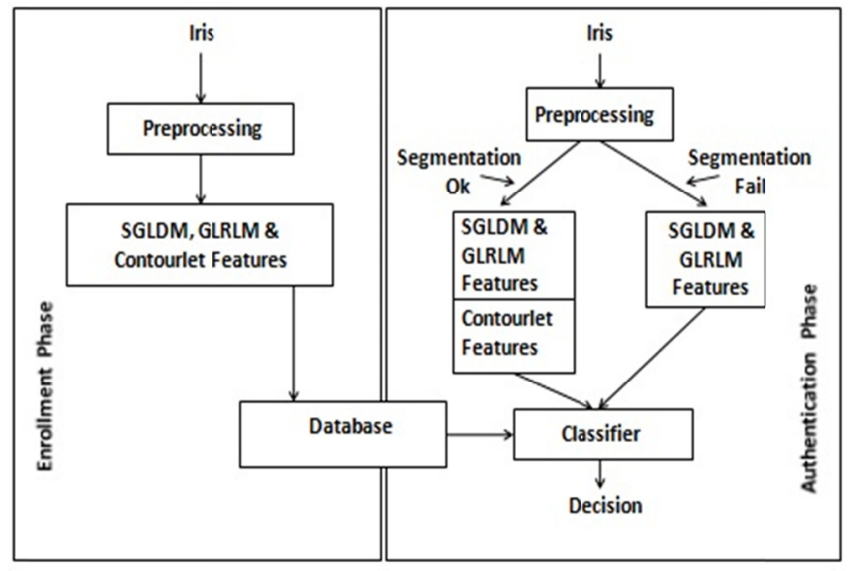

Fig. 3. Proposed method block diagram

\section{A. Literature Survey}

A detailed survey on iris recognition algorithms was presented in [1]. Author in [6] used Fourier transform to detect the fake iris patterns due to the presence of lens which is less reliable. Authors in [10] proved that focus, motion, occlusion and other quality related measures can help fake iris detection.
The effect of both transparent and colored contact lenses on iris recognition was presented in [4, 5, 7]. Authors in [11] applied LBP operator and statistical features for identifying the print attacks in iris recognition in]. Authors in [12] used support vector machines to train the GLCM features of textured lens images. In [13], authors detected textured contact lens by measuring edge sharpness in the iris image, iris texture characterization through iris-textons and co-occurrence matrix. Multi-scale local binary patterns and AdaBoost learning algorithm were used to classify textured lens in [14]. Authors in [15] applied Gaussian smoothed as well as SIFT weighted LBP for the detection of textured lens in iris images. Authors in [7] tested the transparent contact lens images as well as colored lens images using modified LBP. Authors in [16] made use of entropy with LBP to detect liveness. The method proposed here works well on print spoof iris images and iris with contact lens using textural and contourlet features.

\section{IRIS PREPROCESSING AND FEATURE EXTRACTION}

\section{A. Iris Segmentation and Normalization}

In any biometric system, preprocessing of the image and extraction of dissimilar features from it plays an essential role in deciding the recognition rate. In the current study, the circular iris and pupil regions are segmented using integrodifferential operator and the iris region is unwrapped using the method proposed in [17]. As some of the print attacked images from ATVS-Fir database in [8] failed the segmentation step, it is not possible to apply contourlet decomposition on them. Hence they contain only textural features (gradient, SGLDM and GLRLM) and subsequently the images are rejected by the system as 'Fakes'. Images with textured lens and soft lens pass through the segmentation step successfully which are then unwrapped and contourlet features are extracted from them.

\section{B. SGLD Matrix and GLRL Matrix}

There are three parts in the feature vector. The first two parts represent the SGLDM and GLRLM parameters of original eye image and the gradient of eye image. The third part represents the contourlet transform features of normalized iris template. SGLD features and GLRLM features are low level global descriptors derived from the eye image shown in Figure 4. The texture of an image is described by SGLD functions by calculating $p(a, b)$ i.e., how frequently pixel pairs with specific gray levels $a$ and $b$ in a stated spatial relationship occur in an image, creating an SGLD matrix. Once the SGLD matrix is created, then statistical parameters proposed in [18] are derived from it and provide information about the image texture.

$$
\begin{aligned}
& \text { Energy: } \sum_{a, b} p(a, b)^{2} \\
& \text { Contrast: } \sum_{a, b}(a-b)^{2} p(a, b) \\
& \text { Correlation: } \frac{\sum_{a, b} a b p(a, b)-\mu_{x} \mu_{y}}{\sigma_{x} \sigma_{y}} \\
& \text { Sum of squares: Variance } \sum_{a, b}(a-\mu)^{2} p(a, b)
\end{aligned}
$$




$$
\begin{aligned}
& \text { Inverse difference moment: } \sum_{a, b} \frac{1}{(a-b)^{2}} p(a, b) \\
& \text { Entropy: }-\sum_{a, b} p(a, b) \log [p(a, b)]
\end{aligned}
$$

\section{GLRL Matrix}

A GLRL matrix is a set of collinear, consecutive pixels having the same gray level. The length of the run is the number of pixels in the run. Let $p(i, j)$ be the $(i, j)^{\text {th }}$ entry in the GLRL matrix, specifying the number of primitives of all directions having the gray level $i$ and length $j$.

\begin{tabular}{cccc}
1 & 2 & 2 & 4 \\
\hline 1 & 1 & 3 & 4 \\
\hline 2 & 3 & 1 & 4 \\
\hline 4 & 1 & 3 & 1 \\
\hline 2 & 3 & 1 & 1 \\
Input size of $5 \times 4$ & & \\
1 & 2 & 2 & 4 \\
\hline 1 & 1 & 3 & 4 \\
\hline 2 & 3 & 1 & 4 \\
\hline 4 & 1 & 3 & 1 \\
\hline 2 & 3 & 1 & 1 \\
Input size of $5 \times 4$ & &
\end{tabular}

\begin{tabular}{lccccc}
$\mathrm{i}$ & $\mathrm{j}$ & 1 & 2 & 3 & 4 \\
\hline 1 & 2 & 1 & 2 & 1 \\
\hline 2 & 0 & 1 & 2 & 1 \\
\hline 3 & 3 & 0 & 0 & 1 \\
\hline 4 & 1 & 0 & 0 & 0
\end{tabular}

\begin{tabular}{lrrrrr}
$\mathrm{i}$ & $\mathrm{j}$ & 1 & 2 & 3 & 4 \\
\hline 1 & 4 & 2 & 0 & 0 \\
\hline 2 & 2 & 1 & 0 & 0 \\
\hline 3 & 4 & 0 & 0 & 0 \\
\hline 4 & 4 & 0 & 0 & 0 \\
GLRLM of $\operatorname{lize} 4 \times 4$
\end{tabular}

Fig. 4. Row 1: SGLDM example. Row 2: GLRLM example

Texture measures measured from the GLRLM are as follows:

Let $k$ be the number of runs. Then

$$
k=\sum_{i=1}^{N_{g}} \sum_{j=1}^{N_{r}} p(i, j)
$$

where $N_{g}$ is number of gray levels in the image and $N_{r}$ is number of different run lengths.

- Short Runs Emphasis: $R F 1=\frac{1}{k} \sum_{i=1}^{N_{g}} \sum_{j=1}^{N_{r}} \frac{p(i, j)}{j^{2}}$

- Long Runs Emphasis: $R F 2=\frac{1}{k} \sum_{i=1}^{N_{g}} \sum_{j=1}^{N_{r}} j^{2} p(i, j)$

- Gray level non-uniformity: $R F 3=\frac{1}{k} \sum_{i=1}^{N_{g}}\left[\sum_{j=1}^{N_{r}} p(i, j)\right]^{2}$

- Run length non-uniformity: $R F 4=\frac{1}{k} \sum_{j=1}^{N_{r}}\left[\sum_{i=1}^{N_{g}} p(i, j)\right]^{2}$

- Run percentage: $R F 5=(1 / p) \sum_{i=1}^{N_{g}} \sum_{j=1}^{N_{r}} p(i, j)$

where $p$ is the number of runs if all possible runs had a length of one.

\section{Gradient Measure}

One of the most common uses of gradient is edge detection. After gradient images have been computed in horizontal and vertical directions, the pixels with the highest gradient values in the direction of the gradient become edge pixels, and edges may be found in the direction perpendicular to the gradient direction. There is almost no difference in the structure of a soft lens image from a normal iris image but textured lens iris image texture is course and random, which distinctly appears as shown in Figure 4. The iris structure in the soft lens image and the real image is smooth but the additional edge shows the presence of contact lens. The fake iris with print attack (where limbic and pupillary boundaries cannot be localized) has less variance and more uniformity as shown in Figure 5.
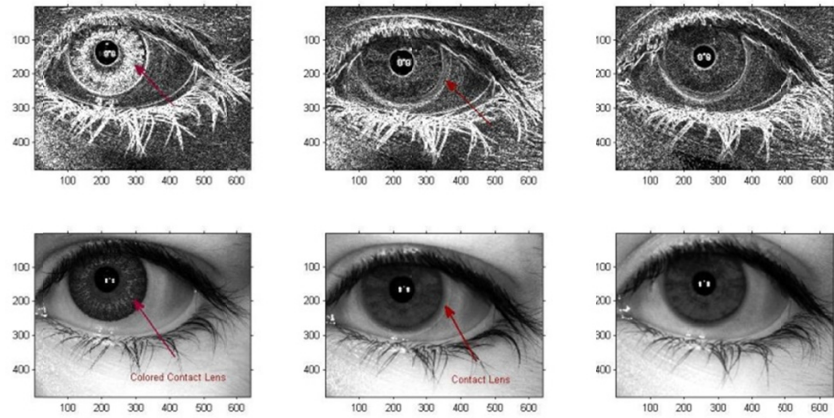

Fig. 5. Row 1: Gradient images of colored, transparent and no lens images. Row 2: Eye images with textured lens, soft lens and no lens.

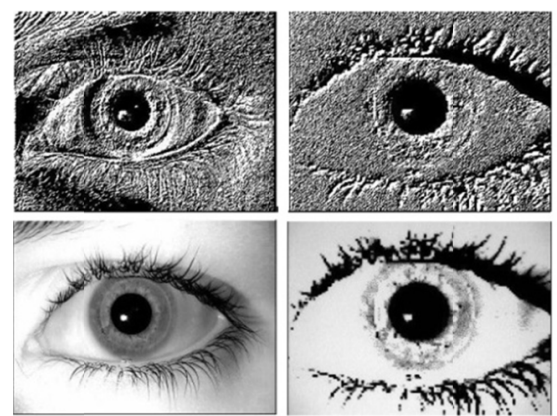

Fig. 6. Row 1: Gradient images of real and fake images. Row 2: ATVSFir real and fake eye images

\section{E. Contourlet Feature Extraction}

Contourlets [21] represent the geometric structures efficiently with little redundancy. Contourlet transform is found as a tool to compensate the limitation of wavelets in handling the geometrical structures that possess directional moments. The double filter bank structure comprising of Laplacian pyramid (LP) and directional filter bank (DFB) is used to catch point discontinuities and linking them in to linear structures respectively. Hence the name pyramidal and directional filter bank (PDFB). It can be noticed that only those contourlets that coincide with location and direction of the image produce significant coefficients. Once the iris image is unwrapped in to $40 \times 240$ template, it is then decomposed in to 16 directions at I level and 4 directions at II level. Statistical parameters are computed for each direction and all are appended to form the feature vector. In the proposed method the iris feature vector is made up of SGLDM, GLRLM of original and gradient images and contourlet coefficients of normalized iris template. Table I summarizes the comparison of existing methods in spoof detection. 
TABLE I. COMPARISON OF EXISTING METHODS IN SPOOF DETECTION

\begin{tabular}{|c|c|c|c|c|}
\hline Method & Attack Nature & Feature & Training/Testing Protocol \\
\hline$[1]]$ & Print spoof & $\begin{array}{c}\text { Statistical features of local and } \\
\text { global eye regions }\end{array}$ & ATVS-Fir: 400 training and 1200 testing \\
\hline$[7]$ & Contact lens & Modified LBP & IIIT-D: \\
\hline$[14]$ & Contact lens & LBP features & $\begin{array}{c}\text { Neural networks fake+6000 live irises for training and 300 fake + } \\
\text { 4000 live irises for testing }\end{array}$ & AdaBoost classifier \\
\hline$[13]$ & Contact lens & Iris textons and Haralick features & CASIA and Bath: Equal train and test samples & SVM classifier \\
\hline$[19]$ & Contact lens & Scale invariant descriptors & $\begin{array}{c}\text { IIIT-D Vista \& IIIT-D Cogent: equal number of training } \\
\text { SVM classifier }\end{array}$ \\
\hline$[16]$ & Print spoof & Entropy with LBP and WP & ATVS-Fir and CASIA-Iris syn databases \\
\hline$[10]$ & Print spoof & $\begin{array}{c}\text { Quality related measures and } \\
\text { SFFS algorithm }\end{array}$ & 200 real images for training and 1400 testing \\
\hline Proposed & $\begin{array}{c}\text { Contact lens } \\
\text { and print spoof }\end{array}$ & $\begin{array}{c}\text { SGLDM, GLRLM features and } \\
\text { contourlet features }\end{array}$ & ATVS-Fir: 500 real for training and 1100 for testing & SVM classifier \\
\hline
\end{tabular}

\section{DATASET PARTITION}

The datasets' description is given in the Table II. Since there are unequal numbers of samples for left and right irises in the IIIT-D databases, we considered 5 samples/each class for the experiment. In ATVS-Fir database 500 samples are used for training and the rest 1100 (300 real +800 fake) samples are used for testing. The eye image and gradient of the eye image are initially subdivided into blocks sized $80 \times 80$ which then form a total of 48 subimages. Feature vectors made up of statistical features defined in section II (B \& C) of SGLDM and GLRLM of subimages are extracted. The size of the textural features will be 1056 (11 features/subimage $\times 48$ subimages $\times$ 2 images). Contourlet decomposition of the iris template generates 20 subimages. The number of frequency domain features is 220 ( 11 features $\times 20$ wedges). The final feature vector size is $1276(1056+220)$. Those fake print attacked images in ATVS-Fir database which fail the segmentation step, will not have contourlet features but contain only the global textural features. At the end of each feature vector the class name is appended facilitating the supervised learning.

TABLE II. DATABASE DETAILS

\begin{tabular}{|c|c|c|c|}
\hline $\begin{array}{c}\text { Database } \\
\text { Parameter }\end{array}$ & IIIT_D Vista & $\begin{array}{c}\text { IIIT-D } \\
\text { Cogent }\end{array}$ & ATVS-FIr \\
\hline Subjects & 101 & 101 & 50 \\
\hline Categories & $\begin{array}{c}\text { Textured lens } \\
\text { Soft lens } \\
\text { Normal }\end{array}$ & $\begin{array}{c}\text { textured lens } \\
\text { Soft lens } \\
\text { Normal }\end{array}$ & $\begin{array}{c}\text { Fake } \\
\text { Real }\end{array}$ \\
\hline Samples/Subject & $\begin{array}{c}\text { Left iris=5 } \\
\text { Right iris=5 }\end{array}$ & $\begin{array}{c}\text { Left iris }=5 \\
\text { Right iris }=5\end{array}$ & $\begin{array}{c}\text { Left iris }=8 \\
\text { Right iris }=8\end{array}$ \\
\hline Classes & 606 & 606 & 200 \\
\hline Total samples & 3030 & 3030 & 1600 \\
\hline Eye image size & $640 \times 480$ & $640 \times 480$ & $640 \times 480$ \\
\hline Train samples & $3 /$ class & $3 /$ class & $5 /$ class (real) \\
\hline Test samples & $2 /$ class & $2 /$ class & $\begin{array}{c}3 / \text { class (real) } \\
+ \text { all fake }\end{array}$ \\
\hline
\end{tabular}

The classification of iris feature vectors is carried out using osusvm package available at [20]. The multiclass SVMs operated in one-against-all approach are tuned with linear support vector classifier kernel. SVMs are trained and tested at train sample:test sample ratio of 3:2 for IIITD databases. The classification of normal, soft lens and textured lens iris images in IIIT-D Vista and Cogent databases is conducted separately as well as mixed. The left and right irises are considered as separate classes in all databases. The train:test sample ratio is 5:3 for ATVS-Fir real database. In this database 500 real iris samples are taken for training and the remaining 1100 (real + fake) samples are considered for testing.

\section{RESULTS AND DISCUSSION}

The experiments are conducted on a PC with Pentium i5, $2.67 \mathrm{GHz}$ processor and 4GB RAM in MATLAB 11.0 environment. The evaluation of the proposed method is done on ATVS-FIr, IIIT-D Vista and IIIT-D Cogent databases. The performance of the proposed method is appraised in terms of correct classification rate (CCR) and compared with existing methods in Table III. The method proposed in [7], obtained $76.83 \%$ and $65.03 \%$. The method described in [19] obtained $89.78 \%$ and $83.88 \%$ and the proposed method achieved better CCR of $95.63 \%$ and $88.83 \%$ on IIITD-Vista and IIITD-Cogent databases respectively. On ATVS-Fir database the proposed method achieved $100 \%$ CCR which is same with [10] and Waleed and Hanna in [16] but better than Chun in [11] which obtained $99.75 \%$. The CCR is less when only either textural or contourlet features are considered but significantly improved when both features are combined. For mixed IIITD database, SVMs are trained with normal and soft lens iris and tested with all three types including textured lens irises. The obtained results are given in Table IV.

TABLE III. \%CRR COMPARISON OF PROPOSED AND EXISTING METHODS

\begin{tabular}{|c|c|c|c|c|c|}
\hline Database & Method & \multicolumn{4}{|c|}{ CRR (\%) } \\
\hline \multirow{4}{*}{$\begin{array}{c}\text { ATVS-Fir } \\
{[8]}\end{array}$} & [11] & \multicolumn{4}{|c|}{99.75} \\
\hline & [16] & \multicolumn{4}{|c|}{100} \\
\hline & [10] & \multicolumn{4}{|c|}{100} \\
\hline & Proposed & \multicolumn{4}{|c|}{100} \\
\hline \multirow{4}{*}{$\begin{array}{c}\text { IIITD-Vista } \\
\text { [9] }\end{array}$} & Lens $^{*}$ & $\mathrm{~N}-\mathrm{N}$ & T-T & S-S & $\mathrm{Avg}^{* *}$ \\
\hline & [19] & 87.77 & 98.59 & 82.99 & 89.78 \\
\hline & [7] & 62.10 & 92.95 & 75.44 & 76.83 \\
\hline & Proposed & 97.64 & 98.85 & 90.40 & 95.63 \\
\hline \multirow{4}{*}{$\begin{array}{c}\text { IIITD-Cogent } \\
\text { [9] }\end{array}$} & Lens ${ }^{*}$ & $\mathrm{~N}-\mathrm{N}$ & T-T & S-S & $\operatorname{Avg}^{* *}$ \\
\hline & [19] & 79.80 & 95.54 & 76.29 & 83.88 \\
\hline & [7] & 65.99 & 80.81 & 48.31 & 65.03 \\
\hline & Proposed & 85.50 & 96.75 & 84.24 & 88.83 \\
\hline
\end{tabular}

Note $^{*}$ N-Normal, S-Soft lens and T-Textured lens. Avg ${ }^{* *}$ : Average 
TABLE IV. PERFORMANCE COMPARISON OF DIFFERENT FEATURES IN \% CCR

\begin{tabular}{|c|c|c|c|c|}
\hline \multirow{2}{*}{ Database } & Lens & $\begin{array}{c}\text { SGLDM } \\
\text { \&GLRLM }\end{array}$ & $\begin{array}{c}\text { Contourlet } \\
\text { Features }\end{array}$ & $\begin{array}{c}\text { Feature } \\
\text { Fusion }\end{array}$ \\
\hline ATVS-Fir [8] & -- & 98.40 & 99.25 & 100 \\
\hline \multirow{4}{*}{ IIITD-Vista [9] } & N-N & 94.68 & 96.56 & 97.64 \\
\cline { 2 - 5 } & T-T & 93.65 & 95.35 & 98.85 \\
\cline { 2 - 5 } & S-S & 86.24 & 87.80 & 90.40 \\
\cline { 2 - 5 } & Mixed & 84.52 & 86.23 & 89.46 \\
\hline \multirow{3}{*}{ IIITD-Cogent [9] } & N-N & 80.40 & 82.78 & 85.50 \\
\cline { 2 - 5 } & T-T & 90.24 & 93.45 & 96.75 \\
\cline { 2 - 5 } & S-S & 79.98 & 81.25 & 84.24 \\
\cline { 2 - 5 } & Mixed & 78.54 & 80.82 & 82.66 \\
\hline
\end{tabular}

Note: Mixed* is trained with normal and soft lens and tested with all 3 types

TABLE V. COMPUTATION TIME FOR EACH BLOCK

\begin{tabular}{|c|c|}
\hline Parameter & Computation time (s) of all databases* \\
\hline Image size & $640 \times 480$ \\
\hline Gradient & 0.12 \\
\hline SGLDM & 0.105 \\
\hline GLRLM & 0.11 \\
\hline Preprocessing & 0.68 \\
\hline Normalization & 0.15 \\
\hline Contourlet features & 0.26 \\
\hline Authentication & 0.012 \\
\hline Total time & 1.437 \\
\hline
\end{tabular}

All databases*: ATVS-Fir, IIITD-Vista and IIITD-Cogent

\section{CONCLUSIONS}

The method proposed in this paper identifies iris images with soft or textured lens and detects print attacks. The set of features consists of optimized textural and frequency domain descriptors. The print spoof images which fail the segmentation step have their contourlet features null. Hence the detection of print attacks is easy during classification. Hence the CCR is $100 \%$ for ATVS-Fir database. There is not much difference between normal and soft lens irises. So the classification rate is high with IIITD databases also. The proposed method is well suited for print spoof as well as lens images.

\section{ACKNOWLEDGMENT}

The ATVS-FIr data set is released by the Biometric Recognition Group, UAM, Madrid, Spain and the IIIT-D Vista and Cogent dataset by IIIT-Delhi, India.

\section{REFERENCES}

[1] K. W. Bowyer, K. Hollingsworth, P. J. Flynn, "Image understanding for iris biometrics: a survey", Computer Vision and Image Understanding, Vol. 110, No. 2, pp. 281-307, 2008

[2] Unique Identification Authority of India, Role of biometric technology in aadhar enrollment, available at https://pdfs.semanticscholar.org /2db1/e82a61e1b30df3853c429f5a669cdb37c41e.pdf, 2012

[3] J. Lee, "Spoofing iris recognition technology with pictures", available at: https://www.biometricupdate.com/201503/spoofing-iris-recognitiontechnology-with-pictures, 2015

[4] J. S. Doyle, P. J. Flynn, K. W. Bowyer, "Automated classification of contact lens type in iris images", 2013 International Conference on Biometrics (ICB), Madrid, Spain, June 7-13, 2013

[5] N. Kohli, D. Yadav, M. Vatsa, R. Singh, "Revisiting iris recognition with color cosmetic contact lenses", 2013 International Conference on Biometrics (ICB), Madrid, Spain, June 7-13, 2013
[6] J. Daugman, "Demodulation by complex-valued wavelets for stochastic pattern recognition", International Journal of Wavelets, Multiresolution and Information Processing, Vol. 1, No. 1, pp. 1-17, 2003

[7] D. Yadav, N. Kohli, J. S. Doyle, R. Singh, M. Vatsa, K. W. Bowyer, "Unravelling the effect of textured contact lenses on iris recognition", IEEE Transactions on Information Forensics and Security, Vol. 9, No. 5, pp. 851-862, 2014

[8] Biometric Recognition Group-ATVS, ATVS-Fir, Fake Iris Images Database, available at: https://atvs.ii.uam.es/atvs/fir_db.html

[9] Image Analysis and Biometrics Lab at in IIIT Delhi, IIITD Contact Lens Database

[10] J. Gallaby, J. Ortiz-Lopez, J. Fierrez, J. Ortega-Garcia, "Iris liveness detection based on quality related features", 5th IAPR International Conference on Biometrics (ICB), New Delhi, India, pp. 271-276, March 29 - April 1, 2012

[11] C.-W. Tan, A. Kumar, "Integrating occular and iris descriptors for fake iris recognition", 2014 International Workshop on Biometrics and Forensics (IWBF), Valletta, Malta, March 27-28, 2014

[12] X. He, S. An, P. Shi, "Statistical texture analysis-based approach for fake iris detection using support vector machines," Lecture Notes in Computer Science, Vol. 4642, pp. 540-546, 2007

[13] Z. Wei, X. Qiu, Z. Sun, T. Tan, "Counterfeit iris detection based on texture analysis," 19th International Conference on Pattern Recognition, Tampa, USA, December 8-11, 2008

[14] Z. He, Z. Sun, T. Tan, Z. Wei, "Efficient iris spoof detection via boosted local binary patterns", Lecture Notes in Computer Science, Vol. 5558, pp. 1080-1090, 2009

[15] H. Zhang, Z. Sun, T. Tan, "Contact lens detection based on weighted LBP”, 20th International Conference on Pattern Recognition, Istanbul, Turkey, pp. 4279-4282, August 23-26, 2010.

[16] W. S. -A. Fathy, H. S. Ali, "Entropy with local binary patterns for efficient iris liveness detection", in: Wireless Personal Communications, Springer Science+Business Media, LLC, 2017

[17] J. Daugman, "How iris recognition works", IEEE Transactions on Circuits and Systems for VideoTechnology, Vol. 14, No. 1, pp. 21-30, 2004

[18] R. M. Haralick, K. Shanmugam, I. Dinstein, "Textural features for image classification", IEEE Transactions on Systems, Man and Cybernetics, Vol. SMC-3, No.6, pp. 610-62, 1973

[19] D. Gragnaniello, G. Poggi, C. Sansone, L. Verdoliva, "Contact lens detection and classification in iris images through scale invariant descriptor", 10th International Conference on Signal-Image Technology and Internet-Based Systems, Marrakech, Morocco, November 23-27, 2014

[20] Support Vector Machines Toolbox, available at https://sourceforge.net/ projects/svm/

[21] M. N. Do, M. Vetterli, "The Contourlet transform: an efficient directional multiresolution image representation", IEEE Transactions on Image Processing, Vol. 14, No. 12, pp. 2091-2106, 2005 